\title{
Contextual effects on encoding and recognition of category members subsumed by two levels of classification
}

\author{
LORRAINE A. LOW \\ Framingham State College, Framingham, Massachusetts
}

\begin{abstract}
The influence of contextual variation of category members during encoding on subsequent recognition of a target member was examined. Triads varying in the relative number of members subsumed by two adjacent levels of categorization were encoded. The superordinate level contained categories such as vehicles; members of this category were taxi, airplane, and subway. The subordinate level contained categories formed by combining the superordinate level with a common attribute, for example, motor vehicles; members of this category included taxi, bus, and limousine. The member that could be contextualized at either level was designated the target-in this example, taxi. During recognition, distractor triads contained a substitute for the target drawn from either of the above levels. Predictions were confirmed for contextual effects during encoding on subsequent false alarm rates.
\end{abstract}

Many studies have been concerned with the role of context in meaning specification (e.g., Anderson \& Ortony, 1975; Barsalou, 1982). However, little attention has been paid to its role in specifying category membership. Tversky's (1977) treatment of the problem is a notable exception. Tversky argued that the meaning of a category member depends on the particular set of members presented and that the set defines the similarity relationship between the presented members. Tulving (1979) similarly stressed the importance of context in instantiating meaning. His encoding specificity hypothesis emphasizes the importance of the meaning at the time of encoding in predicting retrieval strength.

In the present experiment, the role of context in defining meaning of its members was investigated. In this study, as in a previous one (Low \& Roder, 1983), triads of category members were formed from two adjacent levels of classification within the same category. Members of the triad belonged either to a superordinate category such as furniture or to a subordinate category that was formed by combining the superordinate category with a simple attribute, for example, baby furniture. A specific triad from the superordinate level was crib, desk, and stool. From the subordinate class, it was crib, playpen, and highchair. The term crib was designated the target in these examples because it could be included in either the superordinate class, furniture, or the subordinate class, baby furniture. It was expected that variation in the en-

A portion of this research was presented at the annual meeting of the Eastern Psychological Association, Boston, MA, March 1985. I want to express my gratitude to Howard Low for his helpful comments on the manuscript. Mary Lee Cerillo, Nance First, Louetta Keene, Mary Pulaski, and Annepage Wilson assisted in data collection. Requests for reprints should be sent to Lorraine A. Low, Department of Psychology, Framingham State College, Framingham, MA 01701. coded triad would result in concomitant changes in those characteristics associated with the target.

A novel procedure, namely, a recognition test, was used to assess the role of category relatedness in category membership. The recognition substitute was defined by its relationship to the target itself. The substitute was related to the target either at the subordinate level (e.g., bassinet substituted for crib) or at the superordinate level (e.g., couch substituted for crib).

The six encoding conditions in this experiment were defined by the nature of the triad. Five of the six conditions are given in Table 1 . Three conditions were the following: subordinate level (three members from the subordinate category), superordinate level (three members from the superordinate category), and unrelated-total (three members from categories unrelated to each other). Since each member of the unrelated-total triad was from a different category, the triad is not defined at either the superordinate or the subordinate level. In the fourth, the mixed condition, two members of the triad were from the subordinate level and one was from the superordinate level (e.g., crib, playpen, and desk). In the fifth, the mixedcontrol condition (not shown in Table 1 since it contained a different target from the other conditions), the encoding triad was mixed but the target was superordinate (in the above example, the member bassinet could be substituted for the target desk during the recognition phase). Since this triad was mixed during encoding but contained a subordinate triad during recognition, it provided an appropriate control for the differential effects of the level of classification during encoding and during recognition. In the unrelated-partial condition, two members were from the subordinate level category and one was from an unrelated category. This condition provided a comparison with the mixed condition, because it differed from that 
condition only in that the third member was from an unrelated category.

Predictions for the relative contextual effects of the encoded triads on category assignment follow from extant semantic feature theories of category relatedness in taxonomic classifications (Rosch, Mervis, Gray, Johnson, \& Boyes-Braem, 1976). Members of the subordinate class contain many more semantic overlapping features, and are therefore more strongly related to each other, than members of the superordinate class. Members of the category baby furniture, for example, contain many more common features than do members of the general category furniture.

The following predictions are based on the view that these common semantic features, which are present at the time of encoding, define the characteristics associated with the target. Moreover, these characteristics are reflected in the relationship between the target and its corresponding recognition substitute. The strength of this relationship, in turn, should directly influence the magnitude of false alarms during recognition. The first set of predictions involves comparisons of different substitutes in the same encoding triad type. First, in a subordinate-level encoding triad, the subordinate-level substitute should yield many more false alarms than a superordinate-level substitute. For example, in the triad crib, highchair, and playpen, substituting bassinet for crib should result in a higher false-alarm rate than substituting couch. This expectation derives from the general view that members of subordinate-level categories are more strongly related to each other than are members of superordinate-level categories. Second, differences between the subordinateand superordinate-level substitutes should disappear when the encoding triad is from the superordinate level. In the encoding triad crib, desk, and stool, substituting either bassinet or couch for crib should not alter the false-alarm rate. The unrelated-total condition provided a baseline for minimal contextual effects.

A second set of predictions involves comparisons of different encoding conditions, given the same recognitionsubstitute type. Given a substitute from the subordinate level, subordinate-level encoding should produce a greater number of false alarms than superordinate-level encoding. For example, substituting bassinet for crib should yield a greater number of false alarms for the original encoding triad crib, highchair, and playpen (subordinate level) than for the encoding triad crib, couch, and stool (superordinate level). A comparison of the superordinatelevel substitute for the subordinate-level encoding condition with the superordinate-level or unrelated-total encoding condition appears more complex. Theoretically, members of the subordinate-level category contain all the defining features of the members of the superordinatelevel category (Medin \& Smith, 1984). The question is, therefore, whether substituting a super-ordinate-level member for the target will result in the same number of false alarms with a subordinate-level encoding triad as with a superordinate-level encoding triad. The hypothesis was that a subordinate-level encoding triad (e.g., crib, highchair, and playpen at the subordinate level baby furniture) would result in many more weighted features for its member than would a superordinate-level encoding triad (e.g., crib, desk, and stool at the superordinate level furniture). Therefore, substituting couch for crib should yield fewer false alarms for the subordinate-level than for the superordinate-level encoding condition. Hampton (1987) argued for this differential weighting of semantic features of members from conjunctive concepts.

\section{METHOD}

\author{
Subjects \\ Thirty-two Framingham State College student volunteers participated \\ in this experiment.
}

\section{Design and Materials}

Fifty-four sets of category triads were composed for the study. A set consisted of triads for each encoding condition and the given substitute for a particular category. On one-third of the test trials, the encoding triad was presented again (same condition). A representative set is presented in Table 1 . The assignment of a particular set to an experimental condition was random. Triads from any one set were used only once. The design was a 6 (encoding) $\times 3$ (recognition substitute) within-subjects design. A total of 54 triads resulted from the assignment of 3 triads to each of the 18 treatment conditions.

\begin{abstract}
Procedure
Random assignment of treatment conditions to the encoding trials was restricted so that every condition was represented within each block of 18 trials. Treatment conditions over trials were rerandomized for recognition testing with the same restriction. The triads were presented one at a time. Each triad was typed on an index card in capital letters, one word under the other. The position of each member of the triad on the card was randomly determined. The subjects were tested individually, and trials were self-paced. The subjects were instructed to remember each member of the triad and were allotted $5 \mathrm{sec}$ to study each card. Recognition testing followed immediately, with the position of each triad
\end{abstract}

Table 1

Representative Stimulus Set

\begin{tabular}{|c|c|c|c|c|c|c|c|}
\hline \multicolumn{5}{|c|}{ Encoding Triad } & \multirow{2}{*}{\multicolumn{3}{|c|}{ Recognition Substitute }} \\
\hline \multirow{2}{*}{$\begin{array}{c}\text { Subordinate } \\
\text { (baby furniture) }\end{array}$} & \multirow[b]{2}{*}{ Mixed } & \multirow{2}{*}{$\begin{array}{l}\text { Superordinate } \\
\text { (furniture) }\end{array}$} & \multirow[b]{2}{*}{ Unrelated-Partial } & \multirow[b]{2}{*}{ Unrelated-Total } & & & \\
\hline & & & & & Subordinate & Superordinate & Same \\
\hline Crib & Crib & Crib & Crib & Crib & Bassinet & Couch & Crib \\
\hline Playpen & Playpen & Stool & Playpen & Steamshovel & & & \\
\hline Highchair & Desk & Desk & Steamshovel & Horseshoe & & & \\
\hline
\end{tabular}

Note-In this example, the target was crib. Unrelated-Partial = two members from the subordinate-level category and one member from an unrelated category. Unrelated-Total $=$ three members from unrelated categories. 
Table 2

Mean Number of False Alarms for Each Encoding Condition, Recognition Substitute, and Same Condition (Hits)

\begin{tabular}{|c|c|c|c|c|c|c|}
\hline \multirow{2}{*}{$\begin{array}{c}\text { Recognition } \\
\text { Substitute }\end{array}$} & \multicolumn{6}{|c|}{ Encoding Condition } \\
\hline & Subordinate & Mixed & Mixed-Control & Superordinate & Unrelated-Partial & Unrelated-Total \\
\hline Superordinate & 0.34 & 0.78 & 0.59 & 1.00 & 1.03 & 0.44 \\
\hline Subordinate & 1.41 & 1.12 & 0.69 & 1.00 & 1.56 & 0.78 \\
\hline Same (Hits) & 2.84 & 2.50 & & 2.34 & 2.50 & 2.31 \\
\hline
\end{tabular}

member on the card rerandomized. The subjects were asked to look at the card and respond "yes" if every member of the triad had been previously seen, and "no" if any one of the three was different.

\section{RESULTS AND DISCUSSION}

The main response measure was the number of false alarms for each subject in each of the 18 treatment conditions. The mean numbers of false alarms and hits for each treatment condition are presented in Table 2 .

An analysis of variance for the false-alarm rate was performed on the 6 (encoding) $\times 2$ (recognition substitute) within-subjects treatment conditions. The Newman-Keuls test with significance level set at .05 was used to test all specific comparisons. Encoding was statistically significant $[F(5,155)=7.25, p<.01, M S e=0.58]$. Means were 1.30 for the unrelated-partial, 1.00 for the mixed, 1.00 for the superordinate-level, 0.88 for the subordinatelevel, 0.64 for the mixed-control, and 0.61 for the unrelated-total conditions. The unrelated-partial condition produced reliably more false alarms than did the unrelatedtotal, mixed-control, or subordinate level encoding. The mixed and superordinate-level encoding conditions each produced reliably more false alarms than did the unrelatedtotal or mixed-control conditions.

Recognition substitute was statistically significant $[F(1,31)=38.39, p<.01, M S e=0.36]$. Means of 1.09 for the subordinate-level substitute and 0.71 for the superordinate-level substitute revealed that a substitute from the subordinate level during recognition produced a higher level of false alarms than did a substitute from the superordinate level.

The encoding $\times$ recognition substitute interaction was statistically significant $[F(5,155)=4.56, p<.01, M S \mathrm{e}$ $=0.51]$. Specific comparisons yielded higher false-alarm rates for subordinate-level encoding with a subordinatelevel substitute than with a substitute from the superordinate level. These findings support the hypothesis that subordinate-level encoding resulted in strong semantic interrelatedness among members. As a consequence of this strong encoding, there was greater judged similarity to the target of the subordinate-level substitute than of the superordinate-level one. As expected, the means for the superordinate- and subordinate-level recognition substitutes were identical in the superordinate-level encoding condition. When encoding was at the superordinate level, in contrast to the subordinate level, these recognition substitutes were not differentiated. The difference between the two recognition substitutes for the unrelated-total condition was in the expected direction but not statistically reliable. The above findings support the view that semantic context played an important role in influencing similarity judgments.

The differences between recognition substitutes for the unrelated-partial condition were very similar to those described above for the subordinate encoding condition. Since unrelated-partial encoding triads contained two members from the subordinate class and one from an unrelated category, these differences suggest that two members from the subordinate category are sufficient to establish a high degree of semantic interrelatedness.

There were no reliable differences between the recognition substitutes for the mixed encoding condition. This finding is similar to that for superordinate-level encoding. This result suggests that inclusion of one member from the subordinate level, which defined the mixed condition, was sufficient to produce encoding at the superordinate level. The mixed-control condition contained the same treatment combinations as the mixed condition during encoding, and the same as the subordinate-level encoding condition during recognition. Differences between the recognition substitutes were not reliable for this condition. This finding suggests that the reliable differences associated with the nature of the recognition substitute for the subordinate-level encoding condition reflect effects at the time of encoding.

The greater number of false alarms at the subordinate than at the superordinate level for the subordinate-level substitutes was in the expected direction but not reliable. This difference was reliable, however, for the unrelatedpartial condition (see Table 2). Significantly fewer false alarms occurred with the unrelated-total encoding than with the subordinate-level encoding for a subordinate-level substitute. This finding emphasizes the important role that context plays in determining semantic interrelatedness.

The superordinate-level substitute was expected to produce fewer false alarms with encoding at the subordinate than at the superordinate level. It was reasoned that with a subordinate-level context at the time of encoding, a superordinate-level substitute for the target would be highly discriminable. This difference in the predicted direction was reliable (see Table 2). The superordinatelevel substitute produced reliably fewer false alarms than did the same substitute in the unrelated-partial or mixed encoding condition. The number of false alarms was low for the unrelated-total encoding condition as well. How- 
ever, since the number of false alarms for the unrelatedtotal condition was generally low for both types of substitutes, it is reasonable to conclude that the small number of false alarms for the subordinate-level encoding with the superordinate-level substitute did reflect an exclusion of this substitute from the highly related semantic subset.

In order to assess how well the subjects were discriminating the originally presented items from the distractors in each encoding condition, the hit rate was compared with the false-alarm rate for the subordinate-level distractors (recognition change). The rationale for using this recognition substitute was that it represented the tendency to say "yes" to a distractor most closely related to the original target. The mixed-control condition was eliminated from the analysis because the recognition substitute in that condition was different from those in the other conditions. An analysis of variance was performed on the 5 (encoding) $\times 2$ (recognition change) withinsubjects design. Differences associated with encoding were statistically significant $[F(4,124)=6.70, p<.01$, $M S e=0.83]$. The subordinate-level encoding condition produced reliably more combined hits and false alarms than did the superordinate and unrelated-total conditions. The findings suggest that the cirterion for responding "yes" to the re-presentation of the original triads and to the superordinate-level distractors was lower in the subordinate-level encoding condition than in the other two encoding conditions. These findings are consistent with the general theoretical view (Hermann, Frisina, \& Conti, 1978) that the familiarity criterion is lowered with an increase in similarity of the targets to the distractors. Encoding $\times$ recognition change was not statistically significant. Recognition change was highly significant $[F(1,31)$ $=135.43, p<.01, M S e=0.56]$. A much higher rate of hits than false alarms was produced for all conditions. The subjects were discriminating between a representation of the original triad and one containing a substitute from the subordinate level.
In conclusion, the findings strongly support the view that the context is a powerful determinant of the assignment of members to categories. The extent of the relatedness of the target to its corresponding substitute was predicted from the level of the encoding triad. First, the subordinate-level substitute yielded higher false-alarm rates than did a superordinate-level substitute, given a subordinate-level encoding triad. Second, these differences disappeared with the superordinate-level encoding triad. Third, given the superordinate-level substitute, encoding at the subordinate level produced reliably fewer false alarms than did encoding at the superordinate level. All these findings encourage further investigation of the role of context in category definition.

\section{REFERENCES}

Anderson, R. C., \& Ortony, A. (1975). On putting apples into bottles-A problem of polysemy. Cognitive Psychology, 7, 167-180.

Barsalou, L. W. (1982). Context-independent and context-dependent information in concepts. Memory \& Cognition, 10, 82-93.

HAMPTON, J. A. (1987). Inheritance of attributes in natural concept conjunctions. Memory \& Cognition, 15, 55-71.

Hermann, D., Frisina, R. D., \& Conti, G. (1978). Categorization and familiarity in recognition involving a well-memorized list. Journal of Experimental Psychology: Human Learning \& Memory, 4, 428-440.

Low, L. A., \& Roder, B. J. (1983). Semantic relations between encoding and retrieval in cued recall. Memory \& Cognition, 11, 651-659.

Medin, D. L., \& SMITH, E. E. (1984). Concepts and concept formation. Annual Review of Psychology, 35, 113-138.

Rosch, E., Mervis, C. B., Gray, W., Johnson, D., \& BoyesBraEm, P. (1976). Basic objects in natural categories. Cognitive Psychology, 8, 382-439.

TulviNG, E. (1979). Relation between encoding specificity and levels of processing. In L. Cermak \& F. I. M. Craik (Eds.), Levels of processing in human memory. New York: Halsted Press.

Tversky, A. (1977). Features of similarity. Psychological Review, 84, 327-352.

(Manuscript received for publication November 16, 1987.) 